\title{
GeSUT4 mediates sucrose import at the symbiotic interface for carbon allocation of heterotrophic Gastrodia elata (orchidaceae)
}

\author{
Li-Hsuan Ho ${ }^{1}$, Yung-I Lee ${ }^{2}$, Shu-Ying Hsieh ${ }^{1}$, I-Shiuan Lin ${ }^{1}$, Yun-Chien Wu ${ }^{1}$, Han-Yu Ko ${ }^{1}$, \\ Patrick Klemens ${ }^{3}$, Ekkehard Neuhaus ${ }^{3}$, Yi-Min Chen ${ }^{1}$, Tzu-Pi Huang ${ }^{4}$, Chih-Hsin Yeh ${ }^{5}$, \\ and WOEI-JIUN GUO ${ }^{1}$ \\ ${ }^{1}$ National Cheng Kung University \\ ${ }^{2}$ National Museum of Natural Science \\ ${ }^{3}$ University of Kaiserslautern \\ ${ }^{4}$ National Chung Hsing University \\ ${ }^{5}$ Council of Agriculture
}

April 28, 2020

\begin{abstract}
Gastrodia elata, a fully mycoheterotrophic orchid without photosynthetic ability, only grows symbiotically with the fungus Armillaria. The mechanism of carbon distribution in this mycoheterotrophy is unknown. We detected high sucrose concentrations in all stages of Gastrodia tubers, suggesting sucrose may be the major sugar transported between fungus and orchid. Thick symplasm-isolated wall interfaces in colonized and adjacent large cells implied involvement of sucrose importers. Two sucrose transporter (SUT)-like genes, GeSUT4 and GeSUT3, were identified that are highly expressed in young Armillariacolonized tubers. Yeast complementation and radio tracer experiments confirmed that GeSUT4 functioned as a high-affinity sucrose-specific proton-dependent importer. Plasma-membrane/tonoplast localization of GeSUT4-GFP fusions, and high RNA expression of GeSUT4 in symbiotic and large cells indicated that GeSUT4 likely functions in active sucrose transport for intercellular allocation and intracellular homeostasis. Transgenic Arabidopsis overexpressing GeSUT4 had larger leaves but sensitive to excess sucrose and roots were colonized with fewer mutualistic Bacillus, supporting the role of GeSUT4 in regulating sugar allocation. This is not only the first documented carbon import system in a mycoheterotrophic interaction, but also highlights the evolutionary importance of sucrose transporters for regulation of carbon flow in all types of plant-microbe interactions.
\end{abstract}

\section{Summary statement}

Identification of a membrane-localized GeSUT4 sucrose importer revealed sucrose allocation during the mycoheterotrophic symbiosis. Overexpressing GeSUT4 in Arabidopsis promoted leaf growth and suppressed microbe colonization, indicating its role in sucrose uptake to support Gastrodia growth.

\section{Acknowledgements}

We acknowledge the confocal core facility at National Cheng Kung University for fluorescence observations and thanks for Ya-Chi Yu for generating transgenic plants and fusion protein observations. We also appreciate constructive comments from Dr. Peter B Goldsbrough. This work was financially supported by grants from the Ministry of Science and Technology, Taiwan (MOST 104-2311-B-006-004-MY3; MOST 108-2314-B-006 
-077 -MY3) and from the DAAD/NSC PPP program (NSC PPP Germany 106-2911-I-006-506) to WJG and (DAAD PPP Taiwan 57320939) to HEN.

\section{Introduction}

Hundreds of millions of years of co-evolution between plants and fungi have resulted in over $99 \%$ of orchids using partial mycoheterotrophy in which young plants obtain carbon nutrients from fungi prior to the development of green leaves (Yuan et al. 2018). Gastrodia in Orchidaceae represents an unusual fully mycoheterotrophic genus (Leake 1994). For efficient cultivation, G. elata first requires a symbiotic association with Mycena spp. for seed germination and later with Armillaria for tuber formation and sexual reproduction (Kim et al. 2006; Lan, Xu \& Li 1994; Xu \& Fan 2001). Without photosynthetic ability, G.elata requires carbon fromArmillaria fungus and degraded hyphae to complete its $\sim 36$-month life cycle (Zhang \& Li 1980). This unique symbiotic interaction of $G$. elata provides a valuable system to explore how carbon is distributed and allocated in mycoheterotrophic plants.

In symbiotic autotrophic plants, high carbon transfer has been documented in the interface between host roots and the arbuscular mycorrhizae (AM) fungal partner (Vandenkoornhuyse et al. 2007). The morphology is similar to Armillaria during mycoheterotrophic symbiosis. The carbon source has been shown to be key to establish optimal symbiosis (Hennion et al. 2019; Kiers et al. 2011). In particular, soluble sugars, such as sucrose or hexoses, are probably the major form of carbon flow (Garcia, Doidy, Zimmermann, Wipf \& Courty 2016; Gutjahr, Novero, Welham, Wang \& Bonfante 2011). Sugar transporters have been identified that localize on adjacent plant and fungal cells and these may fine-tune sugar exchange ensuring benefits for both partners in this symbiotic relationship (Doidy et al. 2012a; Hennion et al.2019). These transport systems include gene families encoding SUT/SUCs (sucrose transporters), MSTs (monosaccharide transporters) and SWEETs (sugars will eventually be exported transporter) (Doidy et al.2012a; Garcia et al. 2016; Manck-Götzenberger \& Requena

2016). SUT transporters represent the most likely candidate to mediate sucrose transport during plantfungal symbiosis due to increased expression of the SUT genes upon symbiosis (Boldt et al. 2011; Doidy et al. 2012b).

SUTs, encoded by a small gene family, are classified into four major clades (SUT1, SUT2-IIA, SUT2-IIB, SUT4) and mostly function as sucrose/H+ symporters (Doidy et al. 2012b; Julius, Leach, Tran, Mertz \& Braun 2017; Kühn \& Grof 2010). The well- characterized clade 1 includes Arabidopsis At SUC2 (Gottwald, Krysan, Young, Evert

\& Sussman 2000; Srivastava, Ganesan, Ismail \& Ayre 2008) and potatoSt SUT1 (Lemoine, Kühn, Thiele, Delrot \& Frommer 1996), the key sucrose importer that mediates phloem loading in source leaves for longdistance sugar transport (Kühn et al. 2003). In contrast, carriers in clades 2 and 4 mobilize sucrose in sink organs for their growth. For example, OsSUT1 and HvSUT1 (Furbank et al. 2001; Weschke et al. 2000), members of clade 2, are expressed in developing grains to transport sucrose into the endosperm for grain filling (Radchuk et al. 2017; Scofield et al.2002). Potato StSUT4, a clade 4 member, mediates sucrose uptake across the plasma membrane to initiate tuberization and flowering (Chincinska et al. 2008; Weise et al. 2000).

SUT transporters also participate in sucrose allocation between autotrophic plants and symbiotic fungi. Expression of several SUT genes is greatly induced upon inoculation of Medicago and tomato roots with AM fungi (Boldt et al. 2011; Doidy et al. , 2012b). Overexpression of the potato St SUT1 increased AM colonization under high available phosphate, likely due to increased sugar allocation to roots for export to AM fungi (Gabriel-Neumann, Neumann, Leggewie \& George 2011). Similarly, increased sucrose export by silencing expression of a periarbuscular membrane retriever, $S l$ SUT2, promoted mycorrhization in tomato roots (Bitterlich, Krügel, Boldt- Burisch, Franken \& Kühn 2014). These studies illustrate the importance of sucrose 
allocation and the activity of SUT sucrose transporters for optimal plant-fungus symbiosis. A similar sucrose distribution system may also operate in mycoheterotrophic plant cells and require a SUT transport mechanism.

To examine the carbon flow mechanism in mycoheterotrophic plants, we utilized the unique GastrodiaArmillaria symbiotic system to identify two SUT sucrose transporters, GeSUT4 and GeSUT3, which were highly expressed in $G$. elata young symbiotic tubers. Transport analysis and localization experiments revealed that Ge SUT4 functions as a sucrose-specific importer on the plasma membrane and the tonoplast in vivo . Based on functional characterizations in Arabidopsis, we propose that Ge SUT4 participates in fungal derived sucrose import during mycoheterotrophic tuber formation. A model of this process is proposed.

\section{Materials and Methods}

\section{Plant materials and growth conditions}

The mycorrhizal tubers of G. elata were developed from the symbiotic culture with Armillaria strain 2, according to a standard protocol (Yeh, Liao, Huang, Miyajima \& Lee 2017). In brief, timber blocks of Liquidambar formosana were inoculated with PDA cubes containing Armillaria. After one month of culture, the protocorm-like bodies (PLBs) derived from G. elata callus were placed on the colonized timber blocks and grown until they formed juvenile tubers. During 18 months of symbiotic cultivation,

G. elata tubers were harvested at seven stages of development for analysis (Fig. S1). Mature tubers were collected from about 1.5 years of symbiotic cultivation. Tubers were frozen at $-80 \mathrm{oC}$ before analysis.

Arabidopsis thaliana and transgenic Arabidopsis plants used in this study were all Columbia ecotype (Col-0). For all experiments, seeds were surface-sterilized (30\% CLOROX bleach and $0.1 \%$ Triton X100, v/v) and then stratified at 4 oC for three days.

Seeds were germinated and grown on one-half-strength Murashige and Skoog basal salt mixture agar media $\left(\frac{1}{2} \mathrm{MS}\right)$ or potting soils in a growth chamber $\left(22{ }^{\circ} \mathrm{C}\right.$ and $16 / 8 \mathrm{~h}$ light/dark regime, with $\sim 80 \mu \mathrm{mol} \mathrm{m}-2 \mathrm{~s}-1$ illumination) for most experiments (except where indicated). For root growth analysis, seeds were germinated on half-strength MS medium supplemented with $0 \%, 1 \%, 2 \%, 5 \%$ or $7 \%$ sucrose, and grown for $7 \mathrm{~d}$ before imaging. For leaf area measurement, seeds were sown and grown on half-strength MS medium containing 2 $\mu \mathrm{g}$ ml-1 hygromycin for $7 \mathrm{~d}$ under short-day conditions (10/14 h light/dark regime). Seedlings of similar sizes were then transferred to soil and grown for another 3 wks. All leaves were imaged by a scanner. Primary root length and leaf area were quantified using ImageJ.

\section{Sugar content quantification}

Frozen tubers were ground to a powder in liquid nitrogen. Sugar metabolites were then extracted and analyzed as described previously (Hoet al. 2019).

\section{Histological observations}

Tubers were sliced and fixed immediately with $2.5 \%$ glutaraldehyde in $0.1 \mathrm{M}$ phosphate buffer ( $\mathrm{pH} 6.8$ ) at room temperature for $6 \mathrm{~h}$. After fixation, samples were dehydrated using an ethanol series, and embedded in Technovit 7100 (Kulzer \& Co., Hanau, Germany) as described previously (Yeung \& Chan 2015). Sections of $3 \mu \mathrm{m}$ were cut with glass knives on a Reichert-Jung 2040 Autocut rotary microtome. These sections were collected on slides and stained with Periodic acid-Schiff's reaction for total insoluble carbohydrates, and counterstained with either $0.05 \%(\mathrm{w} / \mathrm{v})$ toluidine blue O (TBO) in benzoate buffer for histology or $1 \%$ 
(w/v) amido black 10B in 7\% acetic acid solution for protein staining (Yeung 1984). Images were captured digitally using a CCD camera attached to the microscope.

\section{Transmission electron microscopy}

Developing mycorrhizal tubers were sliced, then fixed with $2 \%$ glutaraldehyde in $0.1 \mathrm{M}$ sodium phosphate buffer (pH 6.8) at $4{ }^{\circ} \mathrm{C}$ overnight. After rinsing three times with fresh phosphate buffer, samples were postfixed with $1 \% \mathrm{OsO} 4$ in the same buffer for $4 \mathrm{~h}$ at room temperature. Samples were dehydrated in a graded acetone series and embedded in Spurr's resin (Electron Microscope Sciences, USA). Ultrathin sections of 70 $\mathrm{nm}$ were cut with a diamond knife on an ultramicrotome Ultracut E (Reichert-Jung, Vienna, Austria). These sections were stained with uranyl acetate and lead citrate. Sections were then examined and photographed using a Philips CM 100 transmission electron microscope at $80 \mathrm{kV}$.

\section{RNA seq analysis}

RNA isolated from tubers at stage 2a was checked for integrity by agarose gel electrophoresis and on the Agilent 2100 Bioanalyzer (Agilent Technologies, CA, USA). The qualified RNA sample was subjected to library construction using the NEBNextUltra RNA Library Prep Kit for Illumina (NEB, USA) following the manufacturer's protocols. A cDNA library was constructed and paired-end sequencing was performed on an Illumina HiSeq 2000 platform. After removing reads containing adapter and low-quality reads, about 54.3 million cleaned reads (i.e., $27.15 \mathrm{M}$ paired- end reads, read length greater than $100 \mathrm{bp}$ ) were obtained. FASTQ sequence files were deposited at the National Center for Biotechnology Information (NCBI) under BioProject PRJNA591090. The clean reads were assembled as unigenes using Trinity method (Grabherr et al. 2011) with optimized k-mer length of 25 into 118,775 contigs.Analysis of mRNA expression levels

Total RNA was isolated from tuber tissues using CTAB extraction buffer combined with RNA purification columns (Ouyang et al. 2014). Full length RNA transcripts were analyzed by RT-PCR using specific primers for the selected genes (GeSUT4-5-BP and

\section{GeSUT4-3-BP for GeSUT4; GeSUT4-5-BP and GeSUT4D-3-BP for GeSUT4D;}

GeSUT3-5-BP and GeSUT3-3-BP for GeSUT3) and 35 cycles of PCR reaction $\left(95{ }^{\circ} \mathrm{C}\right.$ for $30 \mathrm{~s}, 64{ }^{\circ} \mathrm{C}$ for $30 \mathrm{~s}$, and $72^{\circ} \mathrm{C}$ for $120 \mathrm{~s}$ ). RNA of $G$. elata actin 7 (GeAct 7), was also amplified with specific primers (GeAct $7-5$ and GeAct7-3 ) and used as the internal control for quantification. To quantify the expression, total RNA transcripts were reverse-transcribed and gene-specific primers were used for real-time quantitative PCR as described (Ho et al. 2019). Expression of the GeAct 7 gene was used to determine relative expression levels according to the following equation: $1000 \times 2$-(Ctgenex-CtGeAct7) $(\mathrm{Ct}=$ threshold cycle). All sequences of primers and corresponding targets are shown in Table S1.

\section{Analysis of transport activities in yeast}

To express GeSUTs in yeast, 2 distinct GeSUT cDNA sequences (1611 bp for GeSUT4 and 1560 bp for GeSUT3) were amplified using Phusion polymerase (New England Biolabs, USA) using the same genespecific primers used for RT-PCR analysis. The cDNA was first cloned into the pDONR221-f1 vector and subsequently transferred to the pDRf1-GW vector using LR Gateway technology (Grefen et al. 2010). The yeast strains YSL2-1 or EBY4000 were transformed with the resulting constructs (pDRf1- GW-GeSUT4 or pDRf1-GW-GeSUT3) using the LiAc method (Chen et al. 2015). The cDNA of the Arabidopsis H+/sucrose cotransporter AtSUC2, was also amplified and cloned into pDRf1-GW using the same strategy. Transformants were selected and spotted on synthetic deficient media supplemented with or without various concentrations of sugars as described previously (Ho et al.2019). Sequences of primers are provided in Table S1. To analyze 
transport activities with radiotracers, yeast cultures were prepared as described previously (Ho et al. 2019). For a time-dependent uptake assay, uptake buffer

medium containing $2 \mathrm{mM}$ sucrose and $1.5 \mu \mathrm{Ci}[14 \mathrm{C}]$ sucrose $\mathrm{ml}-1$, in $50 \mathrm{mM}$ sodium phosphate (pH 5 ) was prepared. For a concentration-dependent uptake assay, uptake media with increasing sucrose concentrations $(0.02,0.2,1,2,5,10$ and $20 \mathrm{mM}$ sucrose in $50 \mathrm{mM}$ sodium phosphate) and the same molar ratios of $\mu \mathrm{Ci}[14 \mathrm{C}]$ sucrose were prepared. For competition assays, uptake buffer media $(2 \mathrm{mM}$ sucrose with $2 \mu \mathrm{Ci}[14 \mathrm{C}]$ sucrose ml-1) supplemented with $20 \mathrm{mM}$ of various unlabeled sugars (glucose, galactose, fructose, mannose, sucrose, or maltose) were prepared. For analysis under different $\mathrm{pH}$ conditions, uptake buffer media containing $2 \mathrm{mM}$ sucrose with $2 \mu \mathrm{Ci}[14 \mathrm{C}]$ sucrose ml-1 were prepared at different $\mathrm{pH}$ values using $50 \mathrm{mM}$ sodium phosphate buffer. For CCCP treatment, yeast cells were pre-incubated with medium containing $10 \mu \mathrm{M}$ CCCP for 10 min before the uptake analysis. All uptake assays and import of radiotracer were analyzed as described previously (Ho et al. 2019).

\section{Subcellular localization of GFP fusion proteins}

The open reading frame of GeSUT4 without the stop codon was amplified from the pDRf1-GW-GeSUT4 plasmid with specific primers (GeSUT4-5-BP and GeSUT4-3-BP-fusion). The purified cDNA was cloned into pDONR221-f1 and transferred into p2GWF7 by using Gateway technology (Karimi et al. 2007) thereby encoding a GsSUT4-GFP fusion protein. Arabidopsis mesophyll protoplasts were transformed with the p2GWF7-GeSUT4 plasmid as described previously (Ho et al.2019). To localize the plasma membrane and tonoplast, protoplasts were co-transformed with either the membrane marker, At PIP2A-RFP (Nelson, Cai \& Nebenführ 2007) or the tonoplast marker At $\gamma$ TIP-RFP (Jauh, Phillips \& Rogers 1999), always in 1:1 ratio.

\section{In situhybridization}

Developing tubers (8 $\mathrm{mm}$ in length) of $G$. elata were fixed in the FAA solution (18:1:1 of ethanol (50\%), glacial acetic acid and formalin) for $24 \mathrm{~h}$ at $4{ }^{\circ} \mathrm{C}$. Samples were

then dehydrated through an ethanol series and embedded in Histoplast paraffin wax (Thermo Scientific). Sections of $8 \mu \mathrm{m}$ were cut on a Leica RM2235 rotary microtome. Digoxigenin-labeled sense and antisense RNA probes, transcribed from 1053-1353 bp downstream from the start codon, were synthesized following the manufacturer's instructions (Roche Applied Science, USA). The procedures for hybridization and immunological detection of signals with alkaline phosphatase were performed as described previously (Schwarzacher \& Heslop-Harrison 2000).

\section{Generation of transgenic Arabidopsis}

The open reading frame of GeSUT4 cDNA was transferred from pDONR221-f1- GeSUT4 vector to the binary plasmid vector pMDC32 using the LR Gateway technology (Curtis \& Grossniklaus 2003). Arabidopsis plants were then transformed with the resulting construct pMDC32-GeSUT4 and four independent homozygous lines were selected as described previously (Guo et al. 2014). Bacillus subtilis colonization assay Bacillus subtilis strain 168 harboring a GFP reporter (pAD43-25) (Dunn \& Handelsman, 1999) was used for the modified root colonization assays (Allard-Massicotte et al. 2016). In brief, an overnight bacterial culture was inoculated into fresh medium and grown for $3 \mathrm{~h}$ to an OD600 of 0.8. Cells were collected and resuspended at an OD600 of $0.02 \mathrm{in} \mathrm{MSNg}$ inoculation medium (4.3 g of MS, $0.05 \%$ of glycerol and $10 \mu \mathrm{g} \mathrm{ml}-1$ of chloramphenicol in 1liter). 7-d-old Arabidopsis seedlings were harvested, washed with sterile water and transferred to a 24-well plate containing $500 \mu \mathrm{l}$ of the Bacillus solution. After $4 \mathrm{~h}$ of incubation, Arabidopsis 
seedlings were washed with sterilized water and roots were cut off, photographed, and transferred to $1.5 \mathrm{ml}$ Eppondorf tubes containing $1 \mathrm{ml}$ of $0.2 \% \mathrm{NaCl}$. Bacillus cells were detached by sonication. Serial dilutions were performed to determine the number of colony-forming unit (CFU) per $\mathrm{cm}$ of root.

\section{Statistical analysis}

All statistics were performed by Student's t-test and indicated by one $(\mathrm{P}<0.05)$ or two $(\mathrm{P}<0.01)$ asterisks.

\section{Results}

\section{Sucrose is the most abundant sugar that accumulates in Gastrodia tubers}

We first measured the concentration of various sugars at 7 developmental stages of $G$. elata tubers (Fig. S1). Symbiosis withArmillaria is typically established with tubers at stage 2. Tubers in stages 3-5 are actively growing and stage 6 tubers are mature and forming flowering buds. Sucrose was abundant in tubers at all stages, as high as $1 \%$ of the dry mass (Fig. 1). During the early stages of symbiosis (stages 1-2) and in the elongation stages (stages 3-4), the concentration of sucrose was two times higher than those of the hexoses, suggesting that sucrose may be the major form of transported carbon during the early stages of Gastrodia tuber growth.

\section{Distinct apoplastic block for carbon allocation}

To understand how sucrose allocation occurs within the developing tuber, we performed histological and ultrastructural observations on $G$. elata cortical cells in a stage 2a tuber where the basal part had been colonized by Armillaria hyphae (Fig. 2a). Histological observation revealed distinct differentiation in cortical cells (Fig. 2b). Inside the epidermis, there were three to four layers of cortical cells colonized by fungal hyphae (IC in Fig. 2b). In infected cortical cells, Armillaria rhizomorph formed a netting fungal matrix with heavily stained cell wall (Fig. 2b-d). The inner cortical cells, adjacent to infected cells, are enlarged and thus are called large cells. These large cells were filled with numerous starch grains and large vacuoles (Fig. 2b, c). The cell walls were

significantly thickened in large cells when compared to normal cortical cells. At the interface between the infected cells and adjacent large cells, papillary cell wall thickenings in contact with fungal hyphae were observed (white box and arrow in Fig. 2d, e). TEM confirmed the extensive ingrowths of cell wall and plasma membrane toward the cytoplasm of large cells (white arrow in Fig. 2e, f). These histological observations demonstrated distinct apoplastic interfaces located between three layers of cells - fungal cells, infected cells, and inner large cells - suggesting the involvement of membrane transport mechanisms for sucrose allocation in the mycoheterotrophic plant cells.

\section{GeSUTs were highly expressed in G. elata tubers}

To further examine the mechanism of sucrose allocation in $G$. elata, we searched for genes encoding potential sucrose transporters. An RNA-Seq dataset was generated from young mycorrhizal tubers (stage 2a in Fig. S1). All unigenes and contigs were annotated according to the sequence similarity search against the nonredundant protein sequence (nr) database in NCBI using BLASTX algorithm. Contigs 4177 and 5219 were found to encode SUT-like genes with higher FPKM (Fragments Per Kilobase Million) value of 22.77 and 7.83, respectively, suggesting that these two contigs were highly expressed in the stage 2 tubers. Full length sequences of three contigs, 4177-1, 4177-2 and 5219, were then cloned and sequenced. Phylogenetic analysis showed that contigs 4177-2 and 5219 were closely related to the SUT4 and SUT3 clades and thus named GeSUT4 and GeSUT3 (Fig. S2), respectively (Doidy et al. 2012b). Further analysis revealed that contig 4177-1 had a 258 bp C-terminal deletion compared to contig 4177-2 and thus namedGeSUT4D (Fig. 
S3a). GeSUT4D may be an alternative splicing event of GeSUT4. GeSUT4 and GeSUT3 genes encoded proteins with 536 and 520 amino acids, with predicted molecular masses of 57.4 and $55.6 \mathrm{kDa}$, respectively (Fig. S3a). Both GeSUT4 and GeSUT3 proteins have 12 transmembrane domains, typical for SUTs, with both N- and C-termini on the cytoplasmic side (Fig. S3b). The GeSUT4 and GeSUT3 proteins shared only $38.7 \%$ amino acid identity. Interestingly, GeSUT4 shared high levels of amino acid identity with SUT4 clade proteins, such as rice OsSUT2 (Os12g0641400; 58.1\%) and potato StSUT4 (AAG25623.2; 59.8\%), while GeSUT3 shared $64 \%$ identity with OsSUT1 and was grouped into SUT2-IIB (Fig. S2). To examine developmental expression profiles of GeSUT genes, mRNA levels were first analyzed by real-time PCR (Fig. 3a). Two GeSUTgenes were constantly expressed in almost all stages, where expression of GeSUT4 was slightly higher than GeSUT3. To determine if there were possible splicing events, RNA expression was also analyzed by semi-quantitative PCR. Two GeSUT genes exhibited similar patterns where mRNA transcripts were abundant in the early stages of symbiosis (stages 1, 2a and 2b) and elongation stage (stage 4), but lower in mature G. elata tubers (stages 5 and 6) (Fig. 3a). These results suggest that functional GeSUTs are expressed in early developing tubers. To further examine sucrose metabolism in the GastrodiaArmillariainterface, we searched our RNA-Seq dataset to identify genes involved in sucrose metabolism in the apoplasm, such as invertase (INV) (Roitsch \& González 2004) and sucrose synthase (SUS) (Stein \& Granot 2019). By blasting NCBI database, only one contig 5712 (Fig. S4), encoding a potential GeINV gene, was expressed in the developing tubers (Fig. 3c). In contrast, two contigs 4129 and 6657, encoding potential GeSUS genes, were greatly expressed in all stages (Fig. 3c), indicating that GeSUS may be involved in sucrose allocation in the mycoheterotrophic cells.

\section{GeSUT4 complements sucrose uptake deficiency in yeast}

To investigate the transport properties of GeSUTs, cDNAs from the GeSUT4 and

GeSUT3 genes were expressed in the yeast mutant YSL2-1, which cannot grow on medium containing monosaccharides or sucrose due to the lack of functional sugar transporters (Guo et al. 2014). Under acidic growth conditions ( $\mathrm{pH} 5$ ), expression of the Arabidopsis sucrose transporter At SUC2 enables better growth on sucrose- containing medium, especially at $5 \%$ sucrose (Fig. 4a). Expression of Ge SUT4, but not of $G e$ SUT3, complemented the growth deficiency of YSL2-1 on sucrose-containing media to a similar degree as At SUC2 (Fig. 4a). In contrast, growth was not observed on medium buffered at neutral $\mathrm{pH}(\mathrm{pH} 7$ ), even when supplemented with $5 \%$ sucrose. Neither of the Ge SUTs could restore growth of YSL2-1 on hexose-containing media while the expression of a yeast hexose transporter HXT5 complemented the growth deficiency (Fig. S5a). Cells expressing GeSUT s showed no growth inhibition on medium containing the toxic glucose analog 2-deoxyglucose (2-DG) (Fig. S5b), indicating GeSUT s had little capacity to transport hexoses. We also examined yeast cells expressing Ge SUT4D. This protein was unable to restore growth on $5 \%$ sucrose (Fig. 4a, S5), indicating that the C-terminal was necessary for Ge SUT4's transport activity.

\section{GeSUT4 functions as a sucrose-specific high affinity carrier}

To confirm that Ge SUT proteins were functional sucrose transporters, we performed time-dependent sucrose uptake assays using [14C]sucrose into YSL2-1 cells expressing either Ge SUT4 or Ge SUT3. After 5 min more $[14 \mathrm{C}]$ sucrose was detected in yeast cells expressing GeSUT4 compared to GeSUT3 , or the empty vector controls. The uptake of sucrose into GeSUT4 harboring cells increased linearly with time until 30 min and was much greater than observed in yeast cells expressing Ge SUT3 (Fig. 4b). Consequently,

we focused on determining the detailed transport kinetics of GeSUT4. Uptake of [14C]sucrose was observed from as low as $0.01 \mathrm{mM}$ reaching saturation close to $10 \mathrm{mM}$ (Fig. 4c). The deduced $\mathrm{KM}$ and Vmax values of GeSUT4 catalyzed sucrose transport were $2.5 \mathrm{mM}$ and $0.7 \mathrm{nmol} 10^{*} 7$ cells- $1 \mathrm{~h}-1$, respectively (Fig. 4c).

Competition uptake assays using [14C]sucrose and a 10-fold excess of unlabeled sugars were performed to examine transport specificity of Ge SUT4. Consistent with the growth assays (Fig. 4a, S2), only unlabeled sucrose, but none of the hexoses, was able to reduce the import of $[14 \mathrm{C}]$ sucrose (Fig. $4 \mathrm{~d}$ ). The observation 
that a 10-fold excess of maltose impacts [14C]sucrose uptake (Fig. 4d) might be due to a background maltose activity of the strain (Fig. S6) (Chen et al. 2015)

As most SUT proteins function as active, proton-driven sugar carriers (Kühn \& Grof 2010), we further examined the effects of various $\mathrm{pH}$ conditions or the protonophore carbonyl cyanide m-chlorophenyl-hydrazone (CCCP) on sucrose transport activity. Similar to the well characterized proton-coupled sucrose symporter $A t$ SUC2 (Sauer \& Stolz 1994), sucrose uptake activity of Ge SUT1 was decreased to less than 10\% at neutral or alkaline pH conditions, or in the presence of CCCP (Fig. 4e). These results support the assumption that Ge SUT4 functions as a sucrose-specific $\mathrm{H}+$-symporter.

\section{GeSUT4 functions on the plasma membrane and tonoplast}

The subcellular localization of Ge SUT4-GFP was examined in Arabidopsis mesophyll protoplasts. When cotransformed with a plasma membrane marker At PIP2-RFP (Nelson et al. 2007), green fluorescence derived from $G e$ SUT4-GFP appeared in a plasma-membrane ring on the outer side of chloroplasts (shown in red auto- fluorescence, indicated "c" in Fig. 5a). The co-localization ofGe SUT4-GFP signals on the plasma membrane was further confirmed by overlapping with red fluorescence

derived from At PIP2-RFP (Fig. 5a). In some cases, we also observed expression of Ge SUT4-GFP fusions on the inner side of chloroplasts (arrowhead in Fig. 5a). To examine if co-expression of two fusion proteins may result in mis-targeting of Ge SUT4-GFP, cells expressing only Ge SUT4-GFP were stained with the plasma membrane stain, FM4-64. Consistently, co-localization of Ge SUT4-GFP signals on the plasma membrane (asterisks) and inner membrane (arrowhead), likely the tonoplast, was observed (Fig. S7a). To confirm the tonoplast membrane localization, protoplasts were co-transformed with Ge SUT4-GFP and a tonoplast marker, At $\gamma$ TIP-RFP (Jauhet al. 1999). Yellow signals derived from the overlapping expression of both fusion proteins were clearly observed (arrowhead in Fig. 5b). In a lysed protoplast where the tonoplast membrane can separate from the plasma membrane, yellow fluorescence derived from co-localization of Ge SUT4-GFP and At $\gamma$ TIP-RFP fusions was observed (arrowhead in Fig. S7b). These observations demonstrated dual targeting of Ge SUT4 to both plasma membrane and the tonoplast.

\section{GeSUT4 expressed in symbiotic cells and large cells.}

To determine the cell-specific localization of GeSUT4 RNA expression, we performed in situ hybridization using a gene-specific probe. A low level of GeSUT4 RNA was detected in most cells, but extremely strong signals (brown color) were detected in cells surrounded by netting hyphae in Armillaria inoculated cells (Fig. 5C). Distinct signals were also detected in nuclei of large cells adjacent to infected cells.

\section{Ectopic expression of GeSUT4 promotes leaf growth}

Currently it is not possible to produce transgenic Gastrodiaorchid and this limits the experimental approaches available to understand the function of GeSUT4. An alternative is to examine the effect of ectopic expression of GeSUT4 in transgenic Arabidopsis, as has been done with orchid genes (He et al. 2019; Hsu, Huang, Chou \&

Yang 2003) and other sugar transporters from non-model plants(Maet al. 2016; Wang et al. 2018). Arabidopsis plants expressing high levels of GeSUT4 RNA were generated (Fig. 6a). IfGe SUT4 is an active, sucrose-specific importer at the plasma membrane (Fig. 4, 5), accumulation of sucrose in the cytosol due to Ge SUT4 activity may result in sucrose toxicity inhibiting root growth (Lu et al. 2014). As expected, while no consistent growth differences were observed under conditions without sucrose, root growth of GeSUT4 overexpressor lines (OE-12, 13, 19) was significantly reduced compared to Arabidopsis transformed with the empty vector (vector, Fig. 6b). In contrast, when transgenic seedlings were transferred to soil for long-term growth, total leaf area of overexpressor plants was 140\% to 190\% higher than corresponding controls (Fig. 
6c, S8). However, no consistent differences in sugar contents in leaves and roots were measured (data not shown).

\section{GeSUT4 activity limits root colonization with Bacillus subtilis}

To examine whether Ge SUT4 uptake activity could play a role in plant-microbe interactions, we examined the ability of B. subtilis, a plant growth-promoting rhizobacterium, to colonize roots of transgenic Arabidopsis seedlings expressing GeSUT4(Allard-Massicotte et al. 2016). In 9-d-old seedlings, overexpression of GeSUT4 (OE-12,13) significantly decreased colonization efficiency in roots compared to control plants (Col, vector) (Fig. 6d), even when root lengths of all seedlings were similar (Fig. S9).

\section{Discussion}

Sucrose allocation may exist in $G$. elata tuberFungal symbiosis plays a crucial role in nutrient exchange that supports plant communities (Smith \& Read 2008). The Gastrodia-Armillariainteraction represents an

unusual plant adaptation to fully heterotrophy, where the symbiotic fungus provides all the carbon to sustain the plant's growth. How this carbon allocation mechanism developed and functions in this heterotrophic interface is an interesting question in plant biology. Here we show that high levels of sucrose (up to $1 \%$ of dry weight) accumulated in all stages of G. elata tubers (Fig. 1), similar to autotrophic tuber plants such as potato (Schwimmer, Bevenue, Weston \& Potter 1954), indicating that sucrose may be the predominant form of carbohydrate transported into $G$. elata at the symbiotic interface. A similar sucrose flow functions in the heterotrophic relationship between autotrophic- and parasitic plant species (Péronet al. 2017), and at the symbiotic interface between plants and AM fungi (Boldt et al. 2011; Doidy et al. 2012b; Garciaet al. 2016; Gutjahr et al. 2011). Some of this sucrose is likely used for starch biosynthesis given the numerous starch grains observed in infected cortical cells and neighboring large cells (Fig. 2b) (Yeh et al. 2017). Expression of genes involved in starch and sucrose metabolism is also observed in G. elata vegetative tubers (Tsai et al. 2016)(Fig. 3c).

\section{GeSUT4 is a sucrose-specific active importer in G. elata}

We also identified genes encoding sucrose transporters, Ge SUT4 and Ge SUT3, that are expressed in mycoheterotrophic Gastradiacells (Fig. 3a, b). The high expression of GeSUT genes in young and elongating tubers (stages 2A-2B and 4) coincides with high levels of sucrose, but not of glucose, in developing tubers (Fig. 1). As been observed in most plant-fungal interfaces (Doidy et al. 2012a; Garcia et al. 2016; Hennion et al. 2019), distinct expression of GeSUT genes occurred in the absence of any symplastic connections in infected cortical cells and large cells (Fig. 2 and Fig. 5c). The sucrose- specific transport activity of Ge SUT4 was demonstrated by complementation and uptake kinetics in the yeast mutant (Fig. 4). The sensitivity of Ge SUT4-mediated

sucrose import to alkaline $\mathrm{pH}$ and the protonophore CCCP confirmed that this transporter is a protondependent transporter (Fig. 4e), as shown for most characterized SUT4 type carriers (Kühn \& Grof 2010; Reinders, Sivitz \& Ward 2012; Schulz et al. 2011; Weise et al.2000). Considering the high amino acid identity (up to $58 \%$ ) to the Os SUT2 symporter (Eom et al. 2011), it is possible that Ge SUT4 functions as a protonsymporter. The presence of an active uptake mechanism like Ge SUT4 can also be supported by expression of various P-type ATPases in the G. elata genome (Yuan et al. 2018). The absence of Ge SUT3 transport activity in yeast may be due to inappropriate expression in a heterologous system and Ge SUT3 may still be involved in sucrose homeostasis in vivo .

GeSUT4 exhibits a high substrate affinity (KM $\sim 2.5 \mathrm{mM}$ ) for sucrose (Fig. 4c), similar to its close homolog in rice, OsSUT2 (KM $\sim 1.8 \mathrm{mM}$ ) (Eom et al. 2011). The feature nicely fits with the low sucrose contents measured 
in the plant-microbe interfaces (Naher, Radziah, Halimi, Shamsuddin \& Razi 2008). Sucrose transporters characterized in some pathogenic fungal cells also exhibit high affinity to sucrose (Sutton, Henry \& Hall 1999; Wahl, Wippel, Goos, Kamper \& Sauer 2010). The transport properties of GeSUT4 would enable strong competition for carbon nutrients from the symbiotic fungal cells and could provide a strategy to avoid rapid metabolism of sucrose into hexoses during carbon allocation.

\section{GeSUT4 mediates inter- and intra-cellular sucrose allocation}

Distinct accumulation of GeSUT4 transcripts in infected cells (Fig. 5c) and the localization of GeSUT4GFP fusion proteins provide evidence that GeSUT4 physiologically functions at the plasma membrane in symbiotic cells, the primary site for apoplastic sucrose exchange at the mycoheterotrophic interface. GeSUT4transcripts also accumulated in nucleus of large cells (Fig. 5c), which exhibited a distinct apoplastic barrier at the interface facing infected cortical cells (Fig. 2d, e). These observations support the hypothesis that GeSUT4 mediates apoplastic sucrose import between the symbiotic interface as well as during intercellular sugar translocation from the infected cortical cells to the inner large cells. GeSUT4 was also localized on the tonoplast (Fig. 5b, S7b). Similar results have been reported for several other SUT4 proteins, such as AtSUT4,HvSUT2 (Endler et al. 2006), OsSUT2 (Eom et al. 2011) and LjSUT4 (Reinders, Sivitz, Starker, Gantt \& Ward 2008). Such dual-targeting to both the plasma membrane and the endomembrane system has also been observed for NtSUT4 andStSUT4 (Chincinska et al. 2013; Okubo-Kurihara et al. 2010). GeSUT4 may also mediate subcellular sucrose distribution depending on its localization. The involvement of GeSUT4 in multiple transport mechanisms may be related to a marked condensation of the G. elata genome (Yuan et al.2018). Considering that GeSUT4 may also function as a proton-symporter, GeSUT4 may function on the tonoplast to export sucrose from vacuolar lumen to the cytoplasm for intracellular metabolism or further allocation.

\section{Ectopic expression of GeSUT4 affects growth and symbiotic capa- city}

The import activity of Ge SUT4 at the plasma membrane in plantawas supported by experiments showing that overexpression of GeSUT4 in Arabidopsis caused sucrose toxicity in roots and increased leaf expansion due to efficient sucrose import (Fig. 6b, c). The activity of Ge SUT4 also allowed efficient competition for apoplastic sucrose in the symbiotic interface in planta (Fig. 6d), likely by retrieving more apoplastic sucrose that, otherwise, originates from plant roots and serves for Bacillus propagation (Allard- Massicotte et al. 2016; Beauregard, Chai, Vlamakis, Losick \& Kolter 2013). Sucrose

import mediated by GeSUT4 parallels the sucrose import system observed in AM colonized autotrophic plants. Reduced expression of theSlSUT2 active importer, also a SUT4 type transporter, at the symbiotic interface promotes AM mycorrhization in roots (Bitterlichet al. 2014). All these observations highlight the critical involvement of sugar import activity in plant-fungal interactions to fine tune carbon fluxes, both for autotrophic plants and for fully heterotrophic Gastrodia orchids.

\section{The role of GeSUT4 in sucrose allocation}

It has been long believed that the primary source of carbon for Gastrodia is from degradation of fungal cells (Guo \& Xu 1990; Kusano 1911). Based on the high expression of $\beta$-glucosidase and xylanase in symbiotic cortex cells, fungal glycans could be hydrolyzed to derive D-glucose and xylose at the interface of Gastrodia tubers (Yuan et al. 2018) or inside large cells (Guo \& Xu 1990). To prevent their metabolism, the extracellular D-glucose or xylose would be converted to sucrose via plasma membrane localized sucrose synthase 
(Amor, Haigler, Johnson, Wainscott \& Delmer 1995; Noël \& Pontis 2000), which and is also highly expressed in Gastrodia (Fig. 3c) and has been proposed to play a primary role in sucrose translocation(Stein \& Granot 2019; Yao, Gonzales-Vigil \& Mansfield 2019). Based on the results presented here, we propose that plasma-membrane localized Ge SUT4 can actively import apoplastic sucrose to retrieve carbon source when establishing symbiosis with the fungus Armillaria (Fig. 7). This sucrose can also be translocated to the inner large cells along the apoplastic pathway, where Ge SUT4 also functions to import sucrose released from infected cells. When needed, the tonoplast localized Ge SUT4 can also export stored sucrose for cytosolic demand.

\section{Conclusion}

In this study, for the first time, a functional sucrose-specific active transporter, Ge SUT4, was identified that contributes to the carbon flow in a fully mycoheterotrophy orchid,

G. elata. Localization of Ge SUT4 proteins on both the plasma-membrane and tonoplast combined with physiological studies in transgenic Arabidopsis indicate that this sucrose transporter imports carbon from Armillaria fungal cells and also maintains intracellular sucrose homeostasis. Even in the absence of photosynthesis and a vascular translocation system, our studies demonstrate the similarity in sucrose allocation mechanisms between the mycoheterotrophic Gastrodia and autotropic plants, highlighting the evolutionary importance of sucrose transporters in carbon allocation.

Supplemental DataThe following supplemental materials are available. Figure S1. Pictures of developing G. elata tubes.

Figure S2. Phylogenetic tree of SUT transporters. Figure S3. Gene properties of Gastrodia GeSUT genes.

Figure S4. Phylogenetic tree of genes involved in sucrose metabolism from Gastrodia. Figure S5. Transport activity of GeSUT4 to hexoses in yeast. Figure S6. The uptake activity of GeSUT4 to maltose. Figure S7. Localization of GeSUT4 expression. Figure S8. Leaf areas of transgenic Arabidopsis overexpressing GeSUT4. Figure S9. Roots growth of transgenic Arabidopsis overexpressing GeSUT4.

\section{Acknowledgements}

We acknowledge the confocal core facility at National Cheng Kung University for fluorescence observations and thanks for Ya-Chi Yu for generating transgenic plants and fusion protein observations. We also appreciate constructive comments from Dr. Peter B Goldsbrough. This work was financially supported by grants from the Ministry of Science and Technology, Taiwan (MOST 104-2311-B-006-004-MY3; MOST 108-2314-B-006 -077 -MY3) and from the DAAD/NSC PPP program (NSC PPP Germany 106-2911-I-006-506) to WJG and (DAAD PPP Taiwan 57320939) to HEN.

\section{Figure legends}

\section{Figure. 1. Sugar compositions of developing G. elatatubers}

Sugar contents of developing tubers. Metabolites were extracted from freeze-dried samples and analyzed by GC-MS. Results are means \pm SE from three biological samples.

\section{Figure. 2. Cell morphology of G. elata tubers inoculated with Armillaria.}

(a) Developing tubers in association with Armillaria isolates. (b) Histological images of inoculated cortical cells in (a). Red dye indicated formation of thick cell walls. (c) Zoom-in micrograph of fungal matrix in infected cortical cell. (d) Zoom-in image of the interface between the infected cell and the neighboring large 
cell in the white box in (c). (e) Zoom-in image of curving ingrowth of cell wall (white arrows) and plasma membrane $\left(^{*}\right)$ in the white box in (d). (f) Micrograph of transmission electron microscopy showing the protruding of wall ingrowth (white arrows). Abbreviation: f: fungal hyphae; IC: infected cortex cells; N: nucleus; S: starch grain; V: vacuole; WI: wall ingrowth. Scale: 1cm in (a), $100 \mu \mathrm{m}$ in (b), (c), (d); $10 \mu \mathrm{m}$ in (f), (e).

\section{Figure. 3. Expression of GeSUT sucrose transporters in G. elatatubers.}

(a) Real-time PCR analysis of GeSUT expression in developing tubers. Results are means \pm SE from three biological samples. (b) RT-PCR analysis of full length RNA transcripts of GeSUT genes. (c) Expression of genes involved in sucrose metabolism in developing tubers. Expression of a G. elata actin 7 analog, GeACT7 , was used as an expression internal control.

\section{Figure 4. Transport activity of GeSUT4 in yeast.}

(a) Growth assay of YSL2-1 cells expressing GeSUTs. Yeast cells expressing GeSUTs, an empty vector, and the AtSUC2 were seriously diluted and cultured on solid media ( $\mathrm{pH} 5$ or 7 ) supplemented with maltose or sucrose, respectively. Images were taken after incubation at $30{ }^{\circ} \mathrm{C}$ for $4-6$ days. (b) Time-dependent uptake activity of YSL2-1 to sucrose. Yeast cells in (a) were incubated with $1 \mathrm{mM}$ sucrose containing $1.5 \mu \mathrm{Ci}[14 \mathrm{C}]$ sucrose and then cells were harvested at indicated time point for radioactivity measurement. Significant differences from cells expressing the empty vector were determined. (c) Kinetic of GeSUT4 transport. Transformed cells were incubated in media containing indicated concentrations of sucrose with same molar ratio of $[14 \mathrm{C}]$ sucrose. After $10 \mathrm{mins}$, cells were harvested and analyzed for radioactivity. (d) Substrate specificity of GeSUT4 transport activity. Transformed cells were incubated for 10 mins with the control solution with or without 10 times concentrated sugars (Suc, sucrose; Glc, glucose; Gal, galactose; Fru, fructose; Man, mannose; Mal, maltose). Relative uptake rate was calculated compared to cells incubated with the control solution. The background uptake of non-transformed cells (empty) was also shown. Significant differences from cells incubated in the control solution were determined. (e) Effect of $\mathrm{pH}$ and protonophore on GeSUT4 uptake activity. Yeast cells expressing an empty vector, AtSUC2 or GeSUT4 were analyzed. Significant differences from results measured under the $\mathrm{pH} 5$ condition were determined. Results are mean $\pm \mathrm{SE}(\mathrm{n}=3$ in $\mathrm{b}, \mathrm{c}, \mathrm{e}$ or 5 in $\mathrm{d})$.

\section{Figure 5. Localization of GeSUT4 expression.}

(a) Subcellular localization of GeSUT4-GFP fusion proteins in Arabidopsis protoplasts. Fluorescence images derived from GeSUT4-GFP fusions, a membrane marker,

AtPIP2A-RFP fusions, and both were shown. The latter "c" and arrowhead indicate chloroplasts and colocalized signals. (b) Co-localization of Ge SUT4-GFP proteins with a tonoplast marker, AtrTIP-RFP fusions. (c) Cell-specific expression of GeSUT4 analyzed by in situ hybridization. Cross-sections of G. elata tubers in Fig 2a were hybridized with GeSUT4- specific sense and anti-sense probes. Asterisks $\left(^{*}\right)$ indicates locations of strong signals observed. Scales are $10 \mu \mathrm{m}$ in (a) and $100 \mu \mathrm{m}$ in (c).

\section{Fig. 6. Effect of overexpressing $G e S U T 4$ on Arabidopsis growth.}

(a) Transcripts of GeSUT4 in transgenic Arabidopsis. Total mRNA was extracted from leaves of transgenic Arabidopsis overexpressing GeSUT4 (OE-12, 13, 14, 19) or the empty vector (vector). Expression of GeAct'7 was used as a loading control. (b) Effect of excess sucrose on root growth of transgenic Arabidopsis. Seedlings 
were saw and grown on media containing various concentrations of sucrose for $7 \mathrm{~d}$. Primary root lengths were then measured. (c) Leaf areas of transgenic Arabidopsis plants. The whole aerial parts of 4 weekold soil-grown plants were imaged and total leaf areas were analyzed by image J. (d) Colonization ability of Bacillus subtilis on transgenic Arabidopsis roots. Nine-day-old seedlings were incubated with media containing Bacillus suspension. After $4 \mathrm{~h}$, whole roots were collected and CFU were analyzed. Results are mean $\pm \mathrm{SE}(\mathrm{n}=8-12$ in $\mathrm{b}, 7$ in c, and 6 in $\mathrm{d})$. Significant differences from

seedlings expressing the empty vector were determined.Fig 7. A model for GeSUT4 function in $G$. elata. When the juvenile tuber of G. elata establishes symbiosis withArmillaria, monosaccharides derived from degradation of fungus cells are quickly transferred to sucrose via plasma-membrane localized sucrose synthase. To prevent carbon loss, the

plasma-membrane Ge SUT4 symporter at the symbiotic interface mediates actively sucrose uptake into symbiotic cortical cells. Sucrose is then needed to be translocated to inner large cells with thick cell wall barriers, where Ge SUT4 also promotes active sucrose uptake to translocated sucrose into the cytosol of large cells for further distribution. Upon demand, tonoplast-localized Ge SUT4 can also actively export stored sucrose for metabolic need.

\section{Conflict of Interest Statement}

\section{The authors declare that there are no conflicts of interest regarding the publication of this paper.}

\section{References}

Allard-Massicotte R., Tessier L., Lécuyer F., Lakshmanan V., Lucier J.-F., Garneau D., Caudwell L., Vlamakis H., Bais H.P. \& Beauregard P.B. (2016) Bacillus subtilis early colonization of Arabidopsis thaliana roots involves multiple chemotaxis receptors. mBio,7, e01664-01616. Amor Y., Haigler C.H., Johnson S., Wainscott M. \& Delmer D.P. (1995) A membrane- associated form of sucrose synthase and its potential role in synthesis of cellulose and callose in plants. Proceedings of the National Academy of Sciences, 92, 9353-9357. Bitterlich M., Krügel U., Boldt-Burisch K., Franken P. \& Kühn C. (2014) The sucrose transporter SISUT2 from tomato interacts with brassinosteroid functioning and affects arbuscular mycorrhiza formation. The Plant Journal, 78, 877-889. Boldt K., Pörs Y., Haupt B., Bitterlich M., Kühn C., Grimm B. \& Franken P. (2011) Photochemical processes, carbon assimilation and RNA accumulation of sucrose transporter genes in tomato arbuscular mycorrhiza. Journal of Plant Physiology, 168, 1256-1263. Chen H.-Y., Huh J.-H., Yu Y.-C., Ho L.-H., Chen L.-Q., Tholl D., Frommer W.B. \& Guo W.-J. (2015) The Arabidopsis vacuolar sugar transporter SWEET2 limits carbon sequestration from roots and restricts Pythium infection. The Plant Journal, 83, 1046-1058. Chincinska I., Gier K., Krügel U., Liesche J., He H., Grimm B., Harren F., Cristescu S. \& Kuehn C. (2013) Photoperiodic regulation of the sucrose transporter StSUT4 affects the expression of circadian-regulated genes and ethylene production. Frontiers in Plant Science, 4. Chincinska I.A., Liesche J., Krügel U., Michalska J., Geigenberger P., Grimm B. \& Kühn C. (2008) Sucrose transporter StSUT4 from potato affects flowering, tuberization, and shade avoidance response.Plant Physiology, 146, 515-528. Curtis M.D. \& Grossniklaus U. (2003) A gateway cloning vector set for high- throughput functional analysis of genes in planta. Plant Physiology, 133, 462- 469. Doidy J., Grace E., Kühn C., Simon-Plas F.o., Casieri L. \& Wipf D. (2012a) Sugar transporters in plants and in their interactions with fungi. Trends in Plant Science, 17, 413-422. Doidy J., van Tuinen D., Lamotte O., Corneillat M., Alcaraz G.r. \& Wipf D. (2012b) The Medicago truncatula sucrose transporter family: characterization and implication of key members in carbon partitioning towards arbuscular mycorrhizal fungi. Molecular Plant,5, 1346-1358. Dunn A.K. \& Handelsman J. (1999) A vector for promoter trapping inBacillus cereus. Gene, 226, 297-305. Endler A., Meyer S., Schelbert S., Schneider T., Weschke W., Peters S.W., Keller F., Baginsky S., Martinoia E. \& 
Schmidt U.G. (2006) Identification of a vacuolar sucrose transporter in barley and Arabidopsis mesophyll cells by a tonoplast proteomic approach.Plant Physiol, 141, 196-207. Eom J.-S., Cho J.-I., Reinders A., Lee S.-W., Yoo Y., Tuan P.Q., Choi S.-B., Bang G., Park Y.-I., Cho M.-H., Bhoo S.H., An G., Hahn T.-R., Ward J.M. \& Jeon J.-S. (2011) Impaired function of the tonoplast-localized sucrose transporter in rice, OsSUT2, limits the transport of vacuolar reserve sucrose and affects plant growth. Plant Physiology, 157, 109-119. Furbank R.T., Scofield G.N., Hirose T., Wang X.-D., Patrick J.W. \& Offler C.E. (2001) Cellular localization and function of a sucrose transporter OsSUT1 in developing rice grains. Aust J Plant Physiol 28, 11871196. Gabriel-Neumann E., Neumann G., Leggewie G. \& George E. (2011) Constitutive overexpression of the sucrose transporter SoSUT1 in potato plants increases arbuscular mycorrhiza fungal root colonization under high, but not under low, soil phosphorus availability. J Plant Physiol, 9, 911-919. Garcia K., Doidy J., Zimmermann S.D., Wipf D. \& Courty P.-E. (2016) Take a trip through the plant and fungal transportome of mycorrhiza.Trends in Plant Science, 21, 937-950. Gottwald J.R., Krysan P.J., Young J.C., Evert R.F. \& Sussman M.R. (2000) Genetic evidence for the in planta role of phloem-specific plasma membrane sucrose transporters. Proceedings of the National Academy of Sciences of the United States of America, 97, 13979-13984. Grabherr M.G., Haas B.J., Yassour M., Levin J.Z., Thompson D.A., Amit I., Adiconis X., Fan L., Raychowdhury R., Zeng Q., Chen Z., Mauceli E., Hacohen N., Gnirke A., Rhind N., di Palma F., Birren B.W., Nusbaum C., Lindblad-Toh K., Friedman N. \& Regev A. (2011) Full-length transcriptome assembly from RNA-Seq data without a reference genome.Nature biotechnology, 29, 644- 652. Grefen C., Donald N., Hashimoto K., Kudla J., Schumacher K. \& Blatt M.R. (2010) A ubiquitin-10 promoter-based vector set for fluorescent protein tagging facilitates temporal stability and native protein distribution in transient and stable expression studies. The Plant Journal, 64, 355-365. Guo S.-X. \& Xu J.-T. (1990) Studies on the cell ultrastructure in the course of Gastrodia elata digesting Mycena osmundicolalange and Armillaria mellea fr. Acta Mycologica Sinica,9, 218-225. Guo W.-J., Nagy R., Chen H.-Y., Pfrunder S., Yu Y.-C., Santelia D., Frommer W.B. \& Martinoia E. (2014) SWEET17, a facilitative transporter, mediates fructose transport across the tonoplast of Arabidopsis roots and leaves. Plant Physiology, 164, 777-789. Gutjahr C., Novero M., Welham T., Wang T. \& Bonfante P. (2011) Root starch accumulation in response to arbuscular mycorrhizal colonization differs among Lotus japonicus starch mutants. Planta,234, 639. He C., Teixeira da Silva J.A., Wang H., Si C., Zhang M., Zhang X., Li M., Tan J. \& Duan J. (2019) Mining MYB transcription factors from the genomes of orchids (Phalaenopsis and Dendrobium) and characterization of an orchid R2R3-MYB gene involved in water-soluble polysaccharide biosynthesis.Scientific Reports, 9, 13818. Hennion N., Durand M., Vriet C., Doidy J., Maurousset L., Lemoine R. \& Pourtau N. (2019) Sugars en route to the roots. Transport, metabolism and storage within plant roots and towards microorganisms of the rhizosphere. Physiologia Plantarum, 165, 44-57. Ho L.-H., Klemens P.A.W., Neuhaus H.E., Hsieh S.-Y., Ko H.-Y. \& Guo W.-J. (2019) SISWEET1a is involved in glucose import to young leaves in tomato plants. Hsu H.-F., Huang C.-H., Chou L.-T. \& Yang C.-H. (2003) Ectopic expression of an orchid (Oncidium Gower Ramsey) AGL6-like gene promotes flowering by activating flowering time genes in Arabidopsis thaliana. Plant and Cell Physiology, 44, 783-794. Jauh G.-Y., Phillips T.E. \& Rogers J.C. (1999) Tonoplast intrinsic protein isoforms as markers for vacuolar functions. The Plant Cell, 11, 1867-1882. Julius B.T., Leach K.A., Tran T.M., Mertz R.A. \& Braun D.M. (2017) Sugar transporters in plants: new insights and discoveries. Plant and Cell Physiology, 58, 1442-1460. Kühn C., Hajirezaei M.-R., Fernie A.R., Roessner-Tunali U., Czechowski T., Hirner

\& Frommer W.B. (2003) The sucrose transporter StSUT1 localizes to sieve elements in potato tuber phloem and influences tuber physiology and development. Plant Physiology , 131, 102-113.

Karimi M., Depicker A. \& Hilson P. (2007) Recombinational cloning with plant gateway vectors. Plant Physiology, 145, 1144-1154. Kiers E.T., Duhamel M., Beesetty Y., Mensah J.A., Franken O., Verbruggen E., Fellbaum C.R., Kowalchuk G.A., Hart M.M., Bago A., Palmer T.M., West S.A., Vandenkoornhuyse P., Jansa J. \& Bücking. (2011) Reciprocal rewards stabilize cooperation in the mycorrhizal symbiosis.Science, 333, 880-882. Kim Y.-I., Chang K.-J., Ka K.-H., Hur H., Hong I.-P., Shim J.-O., Lee T.-S., Lee J.-Y. \& Lee M.-W. (2006) Seed germination of Gastrodia elata using symbiotic fungi, Mycena osmundicola. Mycobiology,34, 7982. Kühn C. \& Grof C.P.L. (2010) Sucrose transporters of higher plants. Current Opinion in Plant Biology, 13, 287-297. Kusano K. (1911) Gastrodia elata and its symbiotic association with Armillaria mellea. J. Col. 
Agric. Imp. Univ. Tokyo,4, 1-66. Lan J., Xu J. \& Li J. (1994) Study on symbiotic relation between Gastrodia elata and Armillariella mellea by autoradiography. Acta Mycologica Sinica, 13, 219- 222. Leake J.R. (1994) The biology of myco-heterotrophic ('saprophytic') plants. New Phytologist, 127, 171-216. Lu Q., Zhao L., Li D., Hao D., Zhan Y. \& Li W. (2014) A GmRAV ortholog is involved in photoperiod and sucrose control of flowering time in soybean. PloS one, 9, e89145-e89145. Ma Q.-J., Sun M.-H., Liu Y.-J., Lu J., Hu D.-G. \& Hao Y.-J. (2016) Molecular cloning and functional characterization of the apple sucrose transporter gene MdSUT2. Plant Physiology and Biochemistry, 109, 442-451. Manck-Götzenberger J. \& Requena N. (2016) Arbuscular mycorrhiza symbiosis induces a major transcriptional reprogramming of the potato SWEET sugar transporter family. Frontiers in Plant Science,7. Naher U.A., Radziah O., Halimi M.S., Shamsuddin Z.H. \& Razi I.M. (2008) Effect of inoculation on root exudates carbon sugar and amino acids production of different rice varieties. Res. J. Microbiol.,3, 580-587 Nelson B.K., Cai X. \& Nebenführ A. (2007) A multicolored set of in vivo organelle markers for co-localization studies in Arabidopsis and other plants. The Plant Journal, 51, 1126-1136. Noël G.M. \& Pontis H.G. (2000) Involvement of sucrose synthase in sucrose synthesis during mobilization of fructans in dormant Jerusalem artichoke tubers. Plant Science, 159, 191195. Okubo-Kurihara E., Higaki T., Kurihara Y., Kutsuna N., Yamaguchi J. \& Hasezawa S. (2010) Sucrose transporter NtSUT4 from tobacco BY-2 involved in plant cell shape during miniprotoplast culture. J Plant Res. Ouyang K., Li J., Huang H., Que Q., Li P. \& Chen X. (2014) A simple method for RNA isolation from various tissues of the treeNeolamarckia cadamba.Biotechnology, biotechnological equipment, 28, 1008-1013. Péron T., Candat A., Montiel G., Veronesi C., Macherel D., Delavault P. \& Simier P. (2017) New insights into phloem unloading and expression of sucrose transporters in vegetative sinks of the parasitic plant Phelipanche ramosa L. (Pomel). Frontiers in Plant Science,7, 2048-2048. Radchuk V., Riewe D., Peukert M., Matros A., Strickert M., Radchuk R., Weier D., Steinbiß H.-H., Sreenivasulu N., Weschke W. \& Weber H. (2017) Down- regulation of the sucrose transporters HvSUT1 and HvSUT2 affects sucrose homeostasis along its delivery path in barley grains. Journal of Experimental Botany, 68, 4595-4612. Reinders A., Sivitz A., Starker C., Gantt J. \& Ward J. (2008) Functional analysis of LjSUT4, a vacuolar sucrose transporter fromLotus japonicus. Plant Molecular Biology, 68, 289-299. Reinders A., Sivitz A. \& Ward J. (2012) Evolution of plant sucrose uptake transporters (SUTs). Frontiers in Plant Science,3. Roitsch T. \& González M.-C. (2004) Function and regulation of plant invertases: sweet sensations. Trends in Plant Science,9, 606-613. Sauer N. \& Stolz J. (1994) SUC1 and SUC2: two sucrose transporters fromArabidopsis thaliana; expression and characterization in baker's yeast and identification of the histidine-tagged protein. The Plant Journal, 6, 6777. Schulz A., Beyhl D., Marten I., Wormit A., Neuhaus E., Poschet G., Büttner M., Schneider S., Sauer N. \& Hedrich R. (2011) Proton-driven sucrose symport and antiport are provided by the vacuolar transporters SUC4 and TMT1/2. The Plant Journal, 68, 129-136. Schwarzacher T. \& Heslop-Harrison P. (2000) Practical in situhybridization. UK: Bios Scientific Pub. Ltd. Schwimmer S., Bevenue A., Weston W.J. \& Potter A.L. (1954) Potato composition, survey of major and minor sugar and starch components of white potato.Journal of Agricultural and Food Chemistry, 2, 1284-1290. Scofield G.N., Hirose T., Gaudron J.A., Furbank R.T., Upadhyaya N.M. \& Ohsugi R. (2002) Antisense suppression of the rice transporter gene,OsSUT1, leads to impaired grain filling and germination but does not affect photosynthesis. Functional Plant Biology, 29, 815-826. Smith S.E. \& Read, D.J. (2008) Mycorrhizal symbiosis. Academic Press, San Diego. Stein O. \& Granot D. (2019) An overview of sucrose synthases in plants. Frontiers inPlant Science, 10. Sutton P.N., Henry M.J. \& Hall J.L. (1999) Glucose, and not sucrose, is transported from wheat to wheat powdery mildew. Planta,208, 426-430. Tsai C.-C., Wu K.-M., Chiang T.-Y., Huang C.-Y., Chou C.-H., Li S.-J. \& Chiang Y.-C (2016) Comparative transcriptome analysis of Gastrodia elata (Orchidaceae) in response to fungus symbiosis to identify gastrodin biosynthesis-related genes. BMC Genomics, 17, 212. Vandenkoornhuyse P., Mahé S., Ineson P., Staddon P., Ostle N., Cliquet J.-B., Francez A.-J., Fitter A.H. \& Young J.P.W. (2007) Active root-inhabiting microbes identified by rapid incorporation of plant-derived carbon into RNA. Proceedings of the National Academy of Sciences, 104, 16970-16975. Wahl R., Wippel K., Goos S., Kamper J. \& Sauer N. (2010) A novel high-affinity sucrose transporter is required for virulence of the plant pathogen Ustilago maydis. PLoS Biol, 8, e1000303.

Wang L., Yao L., Hao X., Li N., Qian W., Yue C., Ding C., Zeng J., Yang Y. \& Wang 
X. (2018) Tea plant SWEET transporters: expression profiling, sugar transport, and the involvement of CsSWEET16 in modifying cold tolerance in Arabidopsis. Plant Molecular Biology, 96, 577-592.

Weise A., Barker L., Kühn C., Lalonde S., Buschmann H., Frommer W.B. \& Ward

J.M. (2000) A new subfamily of sucrose transporters, SUT4, with low affinity/high capacity localized in enucleate sieve elements of plants. The Plant Cell, 12, 1345-1355. Weschke W., Panitz R., Sauer N., Wang Q., Neubohn B., Weber H. \& Wobus U. (2000) Sucrose transport into barley seeds: molecular characterization of two transporters and implications for seed development and starch accumulation. The Plant Journal,21, 455467. Xu J.T. \& Fan L. (2001) Cytodifferentiation of the seeds (protocorms) and vegetative propagation conns colonized by mycorrhizal fungi.Acta Botanica Sinica, 43, 1003-1010. Yao D., Gonzales-Vigil E. \& Mansfield S.D. (2019) Arabidopsis sucrose synthase localization indicates a primary role in sucrose translocation in phloem.Journal of Experimental Botany, 71, 1858-1869. Yeh C.H., Liao F.S., Huang K.L., Miyajima I. \& Lee Y.I. (2017) An efficient protocol of protocorm-like bodies regeneration from callus cultures of Gastrodia elata blume and the further associations with mycorrhizal fungi. Journal of the Faculty of Agriculture, Kyushu University, 62, 39-46. Yeung E. (1984) Histological and histochemical staining procedures. In Cell culture and somatic cell genetics of plants (eds I.K. Vasil \& I. Vasil), pp. 689-697. Academic Press, Orlando. Yeung E. \& Chan C. (2015) The glycol methacrylate embedding resins-Technovit 7100 and 8100. In Plant microtechniques and protocols (eds E.C.T. Yeung, C. Stasolla, M.J. Sumner \& B.Q. Huang), pp. 67-82. Springer International Publishing, Switzerland. Yuan Y., Jin X., Liu J., Zhao X., Zhou J., Wang X., Wang D., Lai C., Xu W., Huang J., Zha L., Liu D., Ma X., Wang L., Zhou M., Jiang Z., Meng H., Peng H., Liang Y., Li R., Jiang C., Zhao Y., Nan T., Jin Y., Zhan Z., Yang J., Jiang W. \& Huang L. (2018) The Gastrodia elata genome provides insights into plant adaptation to heterotrophy. Nature Communications,9,

1615. Zhang W.J. \& Li B.F. (1980) The biological relationship of Gastrodia elata and Armillaria mellea. Acta Botanica Sinica, 22, 57-62. 


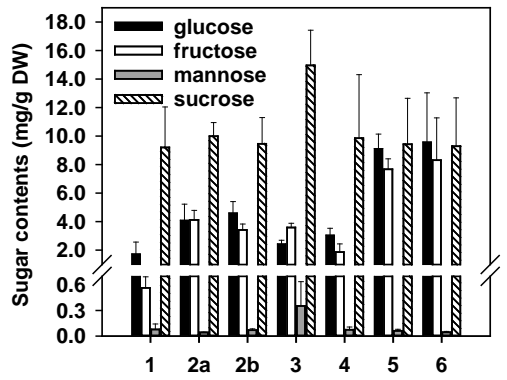

Figure 1. Sugar compositions of developing G. elata tubers

\section{Hosted file}

GeSUT-PCE-Fig-sub1 .pdf available at https://authorea.com/users/313457/articles/443954-gesut4mediates-sucrose-import-at-the-symbiotic-interface-for-carbon-allocation-of-heterotrophicgastrodia-elata-orchidaceae 